\title{
Image Compression Using the Wavelet Transform on Textural Regions of Interest.
}

\author{
D.A. Karras ${ }^{\lambda}$, S.A. Karkanis ${ }^{\star}$ and B.G. Mertzios ${ }^{\star}$ \\ ${ }^{\lambda}$ University of Ioannina, Informatics Dept., Rodu 2, Ano Iliupolis. Athens 16342, Greece, \\ dakarras@cs.uoi.gr \\ ^Univ. of Athens, Dept. of Informatics, TYPA Build., 15784 Athens, Greece, sk@ di.uoa.gr \\ *Democritus Univ.of Thrace, Dept. of Electr.and Comp. Eng., 67100 Xanthi, Greece, \\ mertzios@demokritos.cc.duth.gr
}

\begin{abstract}
This paper suggests a new image compression scheme, using the discrete wavelet transformation (DWT), which is based on attempting to preserve the texturally important image characteristics. The main point of the proposed methodology lies on that, the image is divided into regions of textural significance employing textural descriptors as criteria and fuzzy clustering methodologies. These textural descriptors include cooccurrence matrices based measures and coherence analysis derived features. While rival image compression methodologies utilizing the DWT apply it to the whole original image, the herein presented novel approach involves a more sophisticated scheme in the application of the DWT. More specifically, the DWT is applied separately to each region in which the original image is partitioned and, depending on how it has been texturally clustered, its relative number of the wavelet coefficients to keep is then, determined. Therefore, different compression ratios are applied to the above specified image regions. The reconstruction process of the original image involves the linear combination of its corresponding reconstructed regions. An experimental study is conducted to qualitatively assessing the proposed compression approach. Moreover, this experimental study aims at comparing different textural measures in terms of their results concerning the quality of the reconstructed image.
\end{abstract}

\section{Introduction}

Image compression plays a critical role in telematics applications. It is desired that either single images or sequences of images be transmitted over computer networks at large distances so as that they could be used for a multitude of purposes. For instance, it is necessary that medical images be transmitted so as that reliable, improved and fast medical diagnosis performed by many centers could be facilitated. To this end, image compression is an important research issue. The difficulty, however, in several applications lies on the fact that, while high compression rates are desired, the applicability of the reconstructed images depends on whether some significant characteristics of the original images are preserved after the compression process has been finished.

For instance, in medical image compression applications, diagnosis is effective only when compression techniques preserve all the relevant and important image information needed. This is the case with lossless compression techniques. Lossy compression techniques, on the other hand, are more efficient in terms of storage and transmission needs but there is no warranty that they can preserve the characteristics needed in medical image processing and diagnosis. One important such characteristic of such images is texture. More specifically, texture analysis of these images can lead to very significant results concerning real tissue motion [1] and thus, can result in improved diagnosis.

More generally, however, it is well known that significant visual effects for any kind of image rely on their textural characteristics [2]. The main motivation for the research effort presented in this paper is, precisely, how these important visual effects could be kept without being severely distorted during image compression process. To this end, their efficient coding is necessary. It is well known that such textural characteristics, cannot be captured from images using first order gray scale statistics. More specifically, Julesz concluded, based on experiments on human preattentive perception, that statistics based on second-order distribution of gray levels could be used as discriminators in identifying textures [2]. 
One of the most prominent, along these lines, approaches in texture identification is the one adopted by Haralick [3].

This paper aims at investigating a novel compression scheme of images based on the discrete 2-D wavelet transform having as goal the preservation of their important textural characteristics. At this point it is necessary to briefly discuss about image compression and the wavelet transform. The general idea behind image compression is to remove the redundancy in an image so as to find a more compact representation. A popular method for image compression is the so-called transform coding, which represents the image in a different space than the original, such that the coefficients of the analysis in the basis of the new space are decorrelated. It has been shown that the multiresolution wavelet decomposition is projections onto subspaces spanned by the scaling function basis and the wavelet basis. These projections on the scaling functions basis yield approximations of the signal and the projections on the wavelet basis yield the differences between the approximations of two adjacent levels of resolution. Therefore, the wavelet detail images are decorrelated and can be used for image compression. Indeed, the detail images obtained from the wavelet transform consist of edges in the image. Since there is little correlation among the values on pixels in the edge images, it is easily understood why the wavelet transform is useful in image compression applications. Indeed, image compression is one of the most popular applications of the wavelet transform [4]. However, the most widely accepted contemporary approach for dealing with image compression employing the wavelet transform, is to apply it to the whole image and then, keep its corresponding coefficients which are larger than a predefined threshold. A more sophisticated compression scheme involving the DWT is utilized here in order to achieve preservation of significant image characteristics.

The suggested approach at first attempts to divide the image into subregions according to the distribution of several textural descriptors which belong in two main categories. First, cooccurrence matrices based features and second, coherence analysis based measures are compared at this stage of the proposed methodology. Each subregion is then classified as texturally important or not utilizing fuzzy logic unsupervised techniques. In the sequel, the discrete two-dimensional wavelet transform $[5,6]$ is applied to each such region but the compression ratio imposed depends on the corresponding classification stage decision. The reconstruction process involves the combination, linear in our case, of the reconstructed subregions by utilizing the inverse 2-D discrete wavelet transform. This methodology has been successfully applied, in terms of the trade-off between the preservation of important image features and achievement of high compression ratios, to the well known in the literature Lena picture.

\section{The wavelet compression scheme using textural regions of interest.}

The goal of this paper is to achieve higher compression ratio in images using the two-dimensional DWT more effectively by exploiting image structure characteristics, which are usually unemployed in image compression. More specifically, we aim at exploiting the second order characteristics of images in the design of improved lossy compression systems.

A good measure related to second order image structure is texture [3]. The rationale underlying the proposed compression methodology is that the significance of image regions varies in space. That is, not all image areas are important in describing the spatial probability distribution of its pixel intensities and subsequently in contributing to the visual effects of the image under consideration. A measure of such image region significance can be derived by exploiting textural information. When the textural characteristics in an image region assume high values then, it is reasonable to suppose that the textural information content of this area is very important. Therefore, the image spatial probability distribution can be more precisely derived if a larger number of features describing it is extracted for such an area than for other ones. Thus, if a compression methodology keeps a larger number of coefficients in texturally significant regions than in the other regions then, a much better decompressed image can be finally obtained since its probability distribution can be more accurately restored. In the sequel, the steps involved in the suggested compression scheme are illustrated.

1. The goal of the first stage of the proposed methodology is, therefore, to cluster the image in two classes, namely, in significant and non significant textural regions. To this end, first, the image is raster scanned with sliding windows of $\mathrm{M} \times \mathrm{M}$ dimensions. We have experimented with $256 \times 256$ images and we have found that $M=8$ is a good size for the sliding window. For each such window we perform two kinds of analysis which are subsequently compared in terms of how their associated features contribute to the preservation of textural characteristics, after applying the suggested compression scheme.

2. The first approach herein employed for deriving textural features is based on the cooccurrence matrices [3]. These matrices represent the spatial distribution and the dependence of the gray levels within a local area. Each $(i, j)$ th entry of the matrices, represents the probability of going from one pixel with gray level (i) to another with a gray level (j) under a predefined distance and angle. More matrices are formed for specific spatial distances and predefined angles. From these matrices, sets of statistical measures are computed (called feature vectors) for building different texture models. We have considered four 
angles, namely $0,45,90,135$ as well as a predefined distance of one pixel in the formation of the cooccurrence matrices. Therefore, we have formed four cooccurrence matrices. Due to computational complexity issues regarding cooccurrence matrices analysis a quantization procedure is usually applied to the image under consideration. This paper attempts, also, to evaluate the significance of the factor of quantizing the original image into a lower than 256 number of gray levels for the clustering stage as well as for the reconstruction process involving the inverse 2-D DWT. We have experimented with quantizations of 16, 64 and 256 gray levels. Among the 14 statistical measures, originally proposed by Haralick [3], that are derived from each cooccurrence matrix we have considered only four of them. Namely, angular second moment, correlation, inverse difference moment and entropy.

\section{Energy - Angular Second Moment}

$$
f_{i}=\sum_{i} \sum_{j} p(i, j)^{2}
$$

\section{Correlation}

$$
\boldsymbol{f}_{2}=\frac{\sum_{i=1}^{N_{g}} \sum_{j=1}^{N_{g}}\left(i^{*} j\right) p(i, j)-\mu_{x} \mu_{y}}{\sigma_{x} \sigma_{y}}
$$

Inverse Difference Moment

$$
f_{3}=\sum_{i} \sum_{j} \frac{1}{1+(i-j)} p(i, j)
$$

\section{Entropy}

$$
f_{4}=-\sum_{i} \sum_{j} p(i, j) \log (p(i, j))
$$

3. The second method for deriving textural features employed in the herein comparisons is the coherence analysis of the original images. A brief description of the measures resulting by applying this methodology follows [7]. Let $\theta(x, y)$ denote the estimated orientation angle at point $(x, y)$ and $G(x, y)$ denotes the gradient at the same point. The measure of coherence at the point $W$ is then estimated within a window of size $W$ around the point $\left(x_{0}, y_{0}\right)$, using the projection of the gradient magnitude $G\left(x_{i}, y_{i}\right)$, taken in the direction $\theta\left(x_{i}, y_{i}\right)$, onto the unit vector in the direction $\theta\left(x_{0}, y_{0}\right)$ according to the next formula :

$$
\rho_{0}=G\left(x_{i}, y_{i}\right) \cos \left(\theta\left(x_{0}, y_{0}\right)-\theta\left(x_{i}, y_{i}\right)\right)
$$

The absolute sum of the projections is then, calculated for all $(i, j)$ values within the window $W$. The dimension $\mathrm{w}$ of the sliding window $W$ should be an odd number since it has one central point, namely, $\left(x_{0}, y_{0}\right)$. In this study the value of $\mathrm{w}=5$ has been used.

A better measure of the coherence, which is the one used in this paper, can be obtained by weighting the measure of formula (1) by the gradient magnitude at that point. The new formula for the calculation of the coherence is

$$
\boldsymbol{\rho}=G\left(x_{0}, y_{0}\right) \frac{\sum_{i, j \in W} \| G\left(x_{i}, y_{j}\right) \cos \left(\boldsymbol{\theta}\left(x_{0}, y_{0}\right)-\boldsymbol{\theta}\left(x_{i}, y_{i}\right) \|||\right.}{\sum_{(i, j) \in W} G\left(x_{i}, y_{i}\right)}
$$

Weighting by the gradient magnitude produces higher coherence values at those points that have high contrast information. This coherence measure takes on low values in regions that the texture is rather homogeneous producing by this way textural edges accurately. The variation of this measure is higher in those points that are between the regions with different textural structure.

4. After formulating the patterns, which are onedimensional when coherence is employed, representing each sliding window closely related to measuring textural significance, the next step is the application of a clustering algorithm to them. To this end, we have selected an unsupervised clustering scheme due to its ubiquitous use without specifying the two desired classes. We have experimented with the fuzzy c-means due to its improved properties. After the clustering process has been finished, each image pixel is assigned the label of the class which it belongs to. It could be reasonably considered that the class with the smaller number of members is the texturally important one, when a non-specific image is attempted to be compressed. That is, it is assumed that important image details convey textural information while the rest of the image, in general, does not.

5. When a pattern has been marked as texturally significant, if cooccurrence matrices derived features are used, then, the upper-left point of the corresponding sliding window takes on the label of 255 , otherwise the label of zero. In the same fashion, when coherence analysis based textural features are employed, then, a pixel is labeled with the value of 255 if it has been assigned to the texturally significant class and with zero otherwise. Following this labeling procedure, for each original gray level image a new black-white image IMP results illustrating the significant and non significant partitions.

6. The next step in the suggested compression scheme is to decompose the original image into two images. The first one, G1, is obtained by applying the following formula to each pixel $(\mathrm{x} 0, \mathrm{y} 0)$ of the original image $\mathrm{G} 0$ : $\mathrm{G} 1(\mathrm{x} 0, \mathrm{y} 0)=\operatorname{MIN}(\mathrm{G} 0(\mathrm{x} 0, \mathrm{y} 0), \operatorname{IMPG} 0(\mathrm{x} 0, \mathrm{y} 0))$, 
Where, IMPG0 is the black-white image representing the texturally significant image regions of G0 obtained in the previous step

Similarly, the second image, G2, is the outcome of applying the following formula to each pixel $(\mathrm{x} 0, \mathrm{y} 0)$ of the original image $\mathrm{G} 0$ :

$\mathrm{G} 2(\mathrm{x} 0, \mathrm{y} 0)=\mathrm{MIN}(\mathrm{G} 0(\mathrm{x} 0, \mathrm{y} 0), 255-\mathrm{IMPG} 0(\mathrm{x} 0, \mathrm{y} 0))$

7. Subsequently, the 2-D DWT is applied to G1 and G2 successively. Let us call DWT_G1, DWT_G2 these wavelet transforms respectively. Then, the compression ratio is determined for each one of the DWT_G1, DWT_G2. In all our experiments we have used compression ratios of $60 \%$ for DWT_G1 and $80 \%$ for DWT_G2. DWT_G1' and DWT_G2' are the compressed DWT_G1, DWT_G2 respectively.

8. The final step is image reconstruction. Let us call $\mathrm{G}^{\wedge} 0$ the reconstructed image. This is obtained by the following formula from DWT_G1', DWT_G2':

$$
\begin{gathered}
\mathrm{G}^{\wedge} 0=\mathrm{a}^{*} \mathrm{INV} \text { _2D_DWT(DWT_G1') } \\
\mathrm{b}^{*} \mathrm{INV} \text {-2D_DWT(DWT_G2'), }
\end{gathered}
$$

Where, INV_2D_DWT is the well known inverse transform of the 2-D DWT and a, b are user defined coefficients. In all our experiments we have used $a=1$ and $b=1$. The selection of these coefficients, however, is crucial for dealing with the blocking effects which are expected to appear in the boundaries between the texturally important and non-important regions. Although the results shown in the next section are very promising, more sophisticated schemes than the use of a simple linear combination between

$$
\text { INV_2D_DWT(DWT_G1') and }
$$$$
\text { INV_2D_DWT(DWT_G2'), }
$$

however, are necessary to overcome these problems.

\section{Experimental study and discussion of the results}

The major effort in this work has been set to the direction of defining the significant and non significant textural regions in the image as well as to compare different solutions applicable to such a task. The feature spaces were created on the basis of the statistical measures calculated over the cooccurrence matrices and secondly, on the measure of coherence estimated over the gradient domain of the image. The other major effort of this paper regards the applicability of a wavelet compression scheme that reconstructs the original image from its partitions.

We have tested the proposed approach on the well known image of Lena. The original image is shown in Fig. 1 and the results of clustering, by direct application of the fuzzy c-means, into the two -significant and non significant regions are shown in Figs. 2 to 4 depending on the number of intensity levels used in the estimation of the features. In Fig. 2 the result of the use of 16 intensity levels is shown and in Figs. $3 \& 4$ the results of the use of 64 and 256 levels are respectively shown. The black regions are those characterized as the non significant and the white ones are those characterized as the significant textural regions. From these images it can be noticed that as the levels of intensities increase the more detailed regions are produced. It is a point more or less expected since the information which is carried from the intensity levels contributes to more accurate discrimination of the feature subspaces. Measures calculated over the cooccurrence matrices are enriched in accuracy when larger cooccurrence matrices are used, since the more levels result in more extended such matrices. Concerning the use of the coherence measures produced over the gradient domain of the image, the results are presented in Fig. 5. It is obvious from this figure that the measure of coherence is rather sensitive to the boundaries of the regions of the image. This property is also enhanced from the use of the gradient over which the measure of coherence is estimated. The location of the boundaries is certainly an important task in image processing but these boundaries do not always discriminate among different textural regions. At this point it should be reported that the MATLAB fuzzy toolbox has been employed in all these simulations.

As discussed in length in the previous section, the two original image partitions transformed by the 2-D DWT are then, compressed with different ratios using higher ratio in the non significant region and lower in the significant one. The compressed image is then reconstructed as a linear combination of the two reconstructed parts and the results are presented in Figs. 6-8, depending on the number of gray levels used when the cooccurrence matrices analysis is involved in the first stage of the proposed compression scheme. Thus, 16, 64 and 256 intensity levels correspond to the reconstructed images of fig 6-8 respectively. After experimenting on different ratios, we found that maintaining an analogy of $60 \%$ of the wavelet coefficients for the non significant texture regions and $80 \%$ of the wavelet coefficients for the significant ones leads to the best trade-off between obtaining accepted quality in the reconstructed image and performing maximum compression. Fig. 9 shows the decompressed image using the information of the coherence measure as a criterion for textural significance. Finally, fig. 10 demonstrates the reconstructed image when the compression has been applied to the 2-D DWT of the whole original image and not on its partitions as in the previous figures 6-9. The results obtained above and shown in figures 6-10 illustrate some important trends

1. The larger the number of intensity levels involved in cooccurrence matrices based textural feature extraction, the smoother the reconstructed image and the better in general its quality.

2. However, when the 16 and 64 quantization levels are employed important image details are better preserved 
despite the fact that the image quality is not, in general, better than the one obtained from using 256 levels. This trend is clearly illustrated if one observes Lena's right eye, for instance. Figures 11 to 14 show in pixel detail the reconstruction of Lena's right eye corresponding to different quantization levels.

3. Coherence analysis based textural features lead to worse results concerning reconstructed image quality.

4. Image details are better preserved using the proposed image compression scheme than the usually employed compression schemes involving the 2-D DWT.

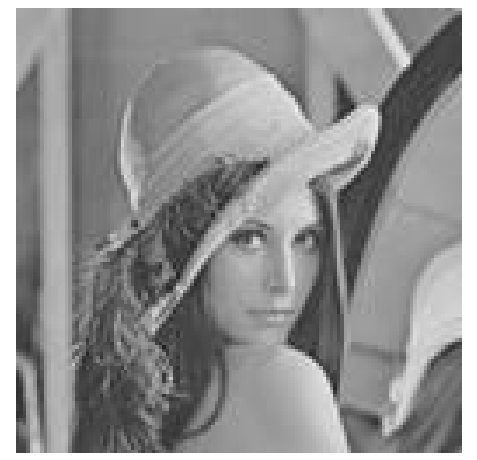

Figure 1. Original image

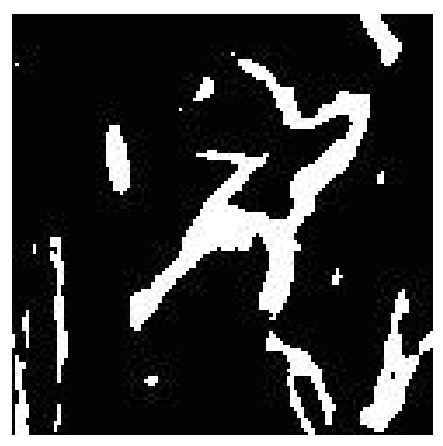

Figure 2. Clustering results using 16 quantisation levels.

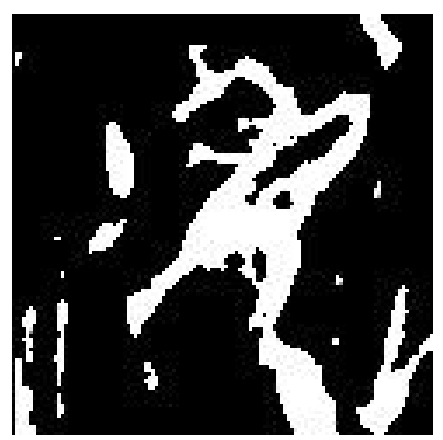

Figure 3. Clustering results using 64 quantisation levels.

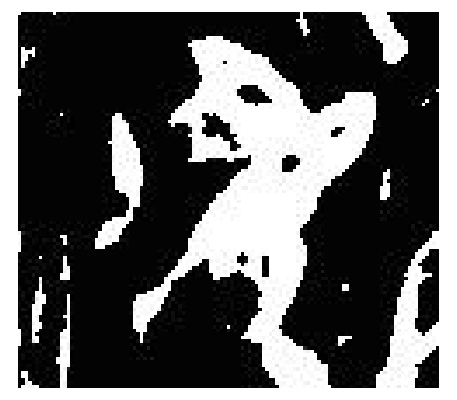

Figure 4. Clustering results using 256 quantisation levels.

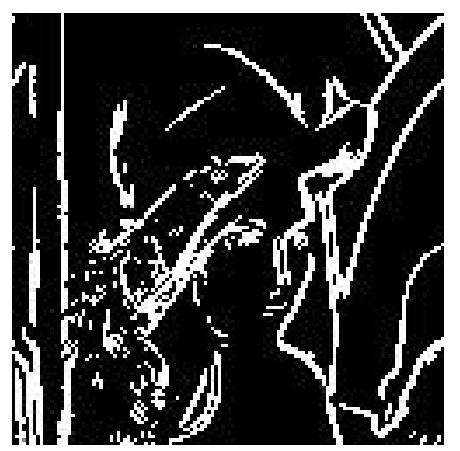

Figure 5. Clustering results using the coherence measure.

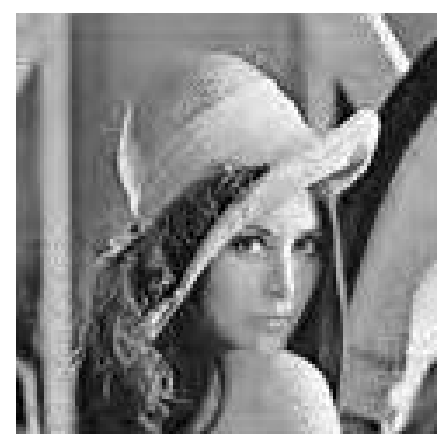

Figure 6. Reconstructed image using 16 quantisation levels.

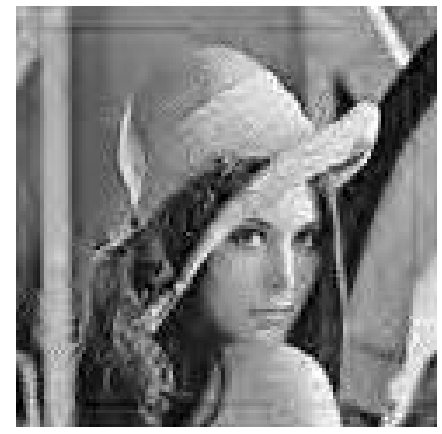

Figure 7. Reconstructed image using 64 quantisation levels. 


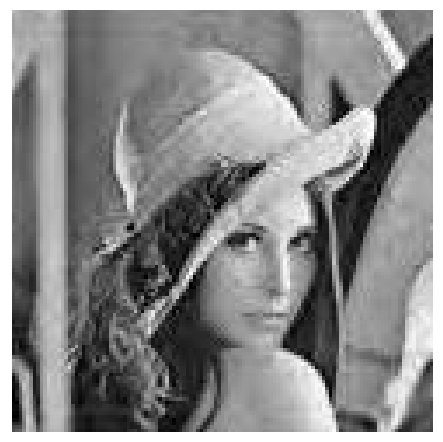

Figure 8. Reconstructed image using 256 quantisation levels.

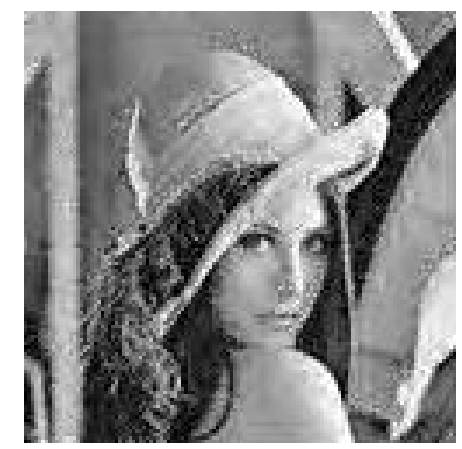

Figure 9. Reconstructed image using the coherence measure.

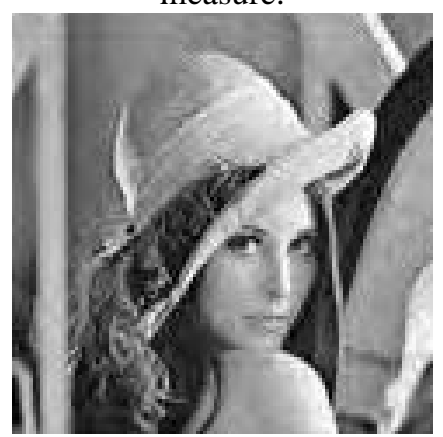

Figure 10. Reconstructed image applying the inverse DWT to the whole image.

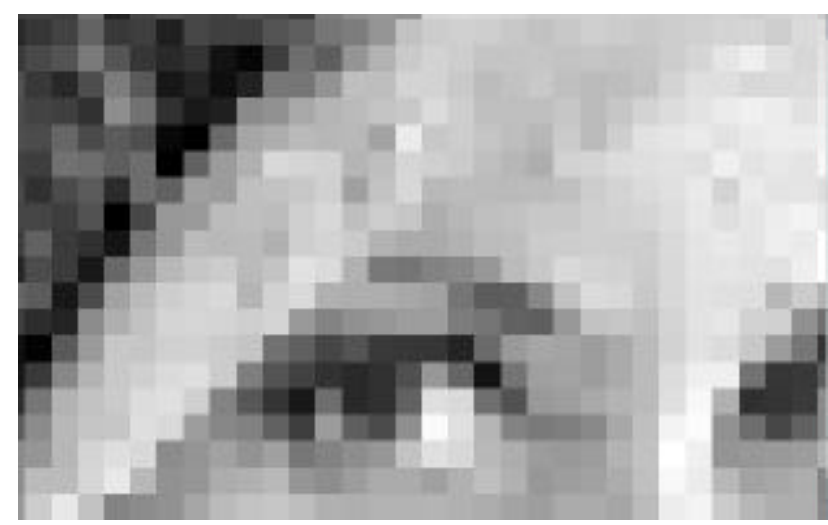

Figure 11. Reconstructed eye region using 16 levels.

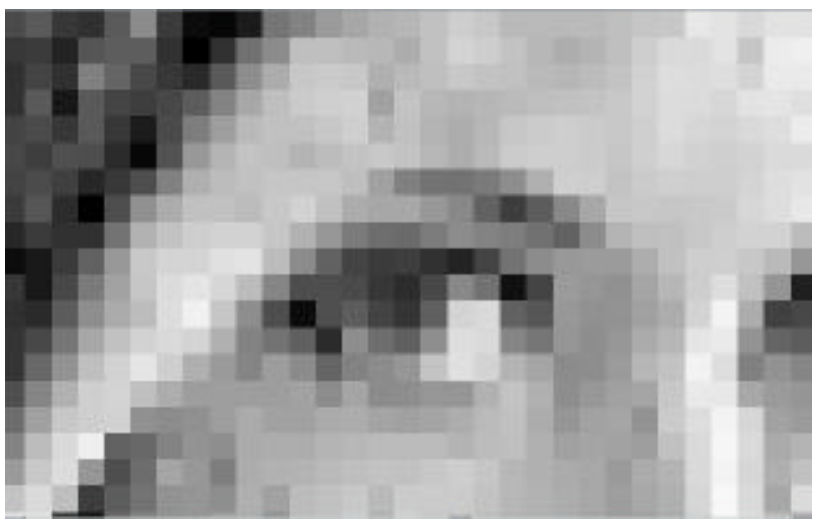

Figure 12. Reconstructed eye region using 64 levels.

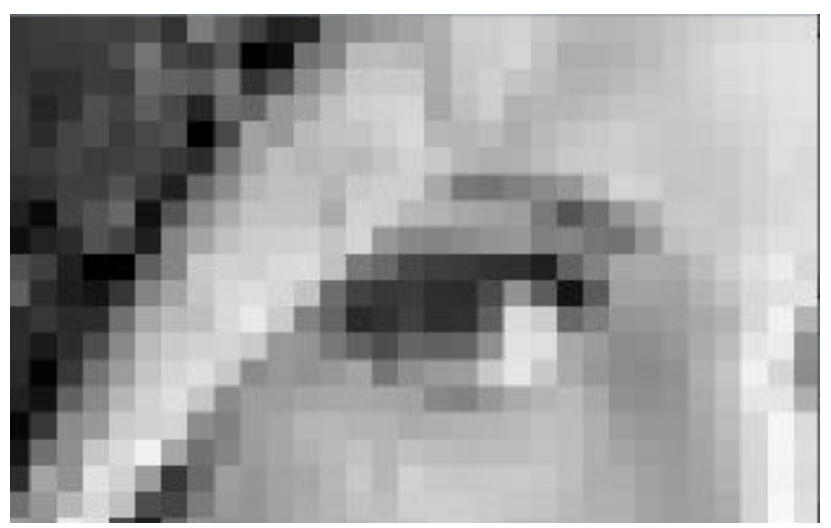

Figure 13. Reconstructed eye region using 256 levels.

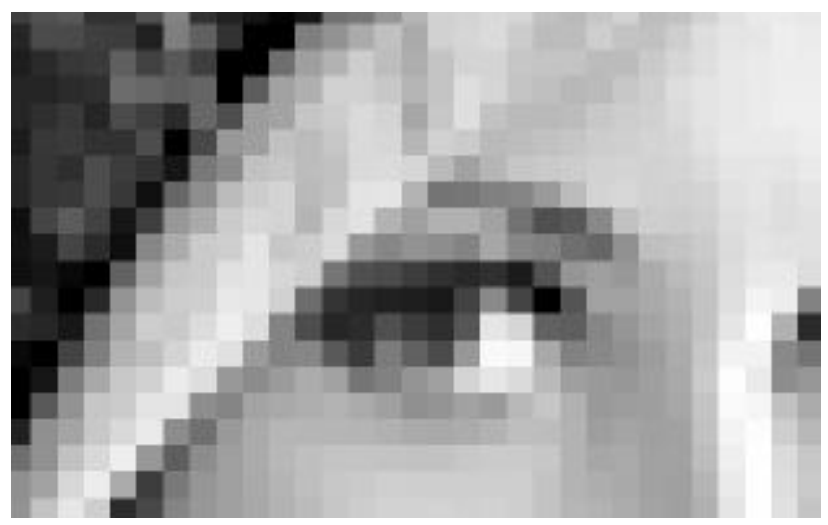

Figure 14. Reconstructed eye region of the original image.

\section{Conclusions}

A novel image compression scheme has been presented based on the 2-d DWT applied to texturally important and non-important image partitions. The promising results obtained concerning reconstructed image quality as well as preservation of significant image details, while, on the 
hand achieving high compression ratios, illustrate that the proposed approach deserves further investigations. The main problem remaining to be dealt with is the elimination of blocking effects in the partitions boundaries, that is the reconstructed image smoothing in these boundaries.

\section{References}

[1] J. Meunier and M. Bertrand, «Ultrasonic texture motion analysis: Theory and Simulation,» IEEE Trans. Med. Imaging, vol. 14, no. 2, pp. 293-300, June 1995.

[2] B. Julesz, «Texton gradients: The texton theory revisited,» Biol. Cybern., vol. 54, pp.245-251, 1986.

[3] R. M. Haralick, «Statistical and structural approaches to texture,»Proc. IEEE, vol. 67, pp. 786-804, 1979.

[4] Poularikas, A. D. (editor),. The Transforms and Applications Handbook. CRC Press and IEEE Press, 1996.

[5] M. Unser, «Texture classification and segmentation using wavelet frames,»IEEE Trans. Image Processing, vol. 4, no. 11, pp. 1549-1560, Nov. 1995.

[6] S. Mallat, «A theory of multiresolution signal decomposition: The wavelet representation,», IEE Trans. Patt. Anal. Machine Intell., vol. 11, no. 7, pp. 674-693, Jul. 1989.

[7] A.Rao «A taxonomy for texture description and identification», Springer-Verlag, New York, 1990 